\title{
SET PROPERTIES DETERMINED BY CONDITIONS ON LINEAR SECTIONS
}

\author{
F. A. VALENTINE
}

Let $R_{n}(n \geqq 2)$ be an $n$-dimensional Euclidean space, and let $S$ be any set of points in $R_{n}$. There exist a number of instances in which the following question has an interesting answer. Suppose a property A holds on each $(n-1)$-dimensional linear section $S_{n-1}{ }^{i}$ of $S$. What additional property B assumed to hold on each section $S_{n-1}{ }^{i}$ will insure that property A holds on $S$ ?

The following terminology is used. A continuum is a compact connected set which may include the degenerate case of a single point. Also compactness includes closure. A generalized continuum is a set which is connected and closed. An $(n-r)$-dimensional linear section of a set $S$ with an $(n-r)$-dimensional Euclidean hyperplane $L_{n-r}$ is defined to be the set $S \cdot L_{n-r}$. A subscript will always designate the dimensionality of the set.

1. Theorems on closed, open and bounded sets. The following theorem illustrates the theory, and plays an important role in a succeeding theorem. It is a case in which condition $B$ is sufficient but not necessary. We shall always assume $n \geqq 2$.

THEOREM 1. Let $S$ be any set in $R_{n}(n \geqq 2)$. If each $(n-1)$-dimensional linear section of $S$ is connected and closed, then $S$ is closed.

Proof. Suppose $S$ is not closed. Then there exists a point $p \notin S$ which is a limit point of $S$. Let $L_{n-1}$ be an $(n-1)$-dimensional hyperplane containing $p$, such that $S \cdot L_{n-1} \neq 0$. Since, by hypothesis, $S_{n-1} \equiv S \cdot L_{n-1}$ is closed, there exists an $(n-1)$-dimensional closed cube $C_{n-1} \subset L_{n-1}$, which contains $p$ in its interior, and for which $C_{n-1} \cdot S_{n-1}=0$. Let $P_{n}$ be an $n$-dimensional hyperprism passing through $C_{n-1}$, and perpendicular to $L_{n-1}$. Since $p$ is a limit point of $S$ which is not in $S$, and since $S_{n-1}$ is closed, there exists a sequence of points $p^{i} \in S \cdot P_{n}$, such that $p^{i} \notin L_{n-1}$, and such that $p^{i} \rightarrow p$ as $i \rightarrow \infty$. Let $L_{n-2}$ be any $(n-2)$-dimensional hyperplane contained in $L_{n-1}$ such that $S \cdot L_{n-2} \neq 0$, and such that $L_{n-2} \cdot C_{n-1}=0$. Then there exists a sequence of hyperplanes $L_{n-1}{ }^{i}$ determined by $L_{n-2}$ and $p^{i}$. By hypothesis each set $S \cdot L_{n-1}{ }^{i}$ is connected. Hence since $p^{i} \in S \cdot L_{n-1}{ }^{i} \cdot P_{n}$, and since any point $q \in S \cdot L_{n-2} \cdot L_{n-1}{ }^{i}$ is not in $P_{n}$, the connectedness of $S \cdot L_{n-1}{ }^{i}$ im-

Presented to the Society, November 24, 1945; received by the editors November 16, 1945, and, in revised form, May 10, 1946. 
plies that $p^{i}$ and $q$ can be joined by a connected subset of $S \cdot L_{n-1}{ }^{i}$ which intersects the boundary of the prism, $B\left(P_{n}\right)$. Hence let $r^{i} \in S \cdot L_{n-1} i \cdot B\left(P_{n}\right)$. Since the set $\left\{r^{i}\right\}$ is infinite, and since the prism $P_{n}$ has a finite number of $(n-1)$-dimensional plane faces, there exist an infinite subset of $\left\{r^{i}\right\}$, namely $\left\{r^{i_{i}}\right\}$, which lie on one face of $P_{n}$. Designate this face by $F_{n-1}{ }^{*}$, so that $r^{i_{i}} \in F_{n-1}{ }^{*} \cdot L_{n-1} i_{i j}$. Furthermore since $p^{i_{j} \rightarrow p}$, as $i_{j} \rightarrow \infty$, the set $\left\{r^{i_{j}}\right\}$ lies on a bounded portion of $F_{n-1}{ }^{*}$. Hence since $L_{n-1} i_{j} \rightarrow L_{n-1}$ as $i_{j} \rightarrow \infty$, the set $\left\{r^{i_{j}}\right\}$ has a limit point $r$ existing in $L_{n-1}$. Since $r^{i_{j}} \in F_{n-1}{ }^{*} \cdot S$, and since by hypothesis $S \cdot L_{n-1} *$ is closed, $r \in S \cdot F_{n-1} *$. Hence $r \in S$. But $r \in F_{n-1} * \cdot L_{n-1} \subset C_{n-1}$, which is a contradiction, since by construction $C_{n-1} \cdot S_{n-1}=0$. Thus the indirect proof is completed, and Theorem 1 is proved.

CoRollary 1.1. If each two-dimensional plane section of $S$ is connected and closed, then $S$ is closed.

CoRollaRY 1.2. If each (n-1)-dimensional linear section of $S$ is a generalized continuum, then $S$ is a generalized continuum.

In Corollary 1.2, the connectedness of $S$ is well known [6, p. 64]. ${ }^{1}$ This second corollary is an illustration where no additional hypotheses $B$ are needed on linear sections in order to guarantee property A on $S$.

THEOREM 2. Let $S$ be any set in $R_{n}(n \geqq 2)$. Suppose that relative to each $(n-1)$-dimensional hyperplane $L_{n-1}$, the set $S \cdot L_{n-1}$ is an open one with a connected complement. Then $S$ is open.

Proof. Let $S_{n-1}{ }^{i}$ be any linear section determined by $L_{n-1}{ }^{i}$. Since $S_{n-1}{ }^{i}$ is open in $L_{n-1}{ }^{i}$, the complement $C\left(S_{n-1}^{i}\right)$ is closed in $L_{n-1}{ }^{i}$. Since each linear set $C\left(S_{n-1}^{i}\right)$ is then connected and closed, Theorem $1 \mathrm{im}$ plies that $C(S)$ is closed. Hence $S$ is open.

CoRollaRy 2.1. Let $S$ be any set in $R_{n}(n \geqq 2)$. If relative to each two-dimensional plane $L_{2}{ }^{i}$, the set $S \cdot L_{2}{ }^{i}$ is an open one with a connected complement, then $S$ is open.

The following theorem is one in which boundedness is the principal property to be established. Here again connectedness plays an important role.

THEOREM 3. Let $S$ be any set in $\mathbb{R}_{n}(n \geqq 2)$. If each $(n-1)$-dimensional linear section of $S$ is bounded and connected, then $S$ is bounded and connected.

\footnotetext{
${ }^{1}$ Numbers in brackets refer to the bibliography at the end of the paper.
} 
Proof. Choose $p \in S$, and let $L_{n-2}$ be a hyperplane containing the point $p$. Consider the family of hyperplanes $L_{n-1}{ }^{\alpha}$ passing through $L_{n-2}$. Let $S_{n}{ }^{m}(m=1,2, \cdots)$ be a set of spheres with centers at $p$ of radii $m$.

Suppose that $S$ is unbounded. Then there exists a sequence of points $x^{i} \in S(i=1,2, \ldots)$ such that the distance $\delta\left(p, x^{i}\right) \rightarrow \infty$ as $i \rightarrow \infty$. Let $L_{n-1}{ }^{i}$ designate the member of $L_{n-1}{ }^{\alpha}$ for which $x^{i} \in L_{n-1}{ }^{i}$. Since $p \in S \cdot L_{n-1} i \cdot S_{n}{ }^{m}$, and since, for any fixed value of $m, x^{i} \in S \cdot L_{n-1}{ }^{i}-S_{n}{ }^{m}$ (for sufficiently large values of $i$ ), the connectedness of $S \cdot L_{n-1}{ }^{i}$ implies that $p$ and $x^{i}$ can be joined by a connected subset of $S \cdot L_{n-1}{ }^{i}$ which intersects the boundary of $S_{n}{ }^{m}$, $B\left(\mathcal{S}_{n}{ }^{m}\right)$. Choose $y^{m, i} \in S \cdot L_{n-1}{ }^{i} \cdot B\left(\mathcal{S}_{n}{ }^{m}\right)$. Since $B\left(\mathcal{S}_{n}{ }^{m}\right)$ is compact, there exists a convergent subsequence $\left\{y^{m, i_{j}}\right\}$ which converges to a point $y^{m} \in \mathcal{S}_{n}{ }^{m}$, such that for the corresponding points $x^{i}, \delta\left(p, x^{i_{i}}\right) \rightarrow \infty$ as $i_{j} \rightarrow \infty$. Without loss of generality designate $L_{n-1}{ }^{0}$ to be the member of $L_{n-1}{ }^{\alpha}$ such that $y^{m} \in L_{n-1}{ }^{0}$. There exists an integer $N$ such that when $i_{j}>N, x^{i_{i}} \in S \cdot L_{n-1} i_{j}-S_{n}{ }^{m+1}$, and such that $\delta\left(p, x^{i_{i}}\right) \rightarrow \infty$ as $i_{j} \rightarrow \infty$. Hence by the connectedness of $S \cdot L_{n-1} i^{i}$, there exist points $y^{m+1, i_{i}} \in S \cdot L_{n-1}{ }^{i j} \cdot B\left(S_{n}{ }^{m+1}\right)$. Since $y^{m, i_{j} \rightarrow y^{m}}$, and since $L_{n-1}{ }^{i j} \rightarrow L_{n-1}{ }^{0}$ as $i_{j} \rightarrow \infty$, there exists a convergent subsequence of $\left\{y^{m+1, i_{j}}\right\}$ which converges to a point $y^{m+1} \in \bar{S} \cdot L_{n-1}{ }^{0} \cdot S_{n}{ }^{m+1}$. Since the radius of $S_{n}{ }^{m}$ is $m$, by induction it follows there exists a sequence $y^{m} \in \bar{S} \cdot L_{n-1}{ }^{0}$, such that $\delta\left(p, y^{m}\right) \rightarrow \infty$ as $m \rightarrow \infty$.

If $S \subset L_{n-1}{ }^{0}$, Theorem 3 is obviously true. Hence, suppose $q \in S$ $-L_{n-1}{ }^{0}$. Since $L_{n-1}{ }^{0}$ divides $R_{n}$ into two half-spaces, namely $R_{n}{ }^{+}$ and $R_{n}{ }^{-}$, suppose without loss of generality that $q \in R_{n}{ }^{+}$. Choose a hyperplane $L_{n-1}+\subset R_{n}{ }^{+}$so that $L_{n-1}{ }^{+}$is parallel to $L_{n-1}{ }^{0}$, and such that $q$ is not on $L_{n-1}+$ or between $L_{n-1}{ }^{+}$and $L_{n-1}{ }^{0}$. Since $y^{m} \in \bar{S} \cdot L_{n-1}{ }^{0}$ there exists in any neighborhood of $y^{m}$ a point $p^{m} \in S$, such that $p^{m}$ and $q$ are on opposite sides of $L_{n-1}{ }^{+}$. Join $p^{m}$ and $q$ by a line $L_{1}{ }^{*}$. Hence $L_{1}{ }^{*} \cdot L_{n-1}{ }^{+} \equiv r^{m}$ exists. Let $L_{n-2}{ }^{*} C L_{n-1}{ }^{+}$be a hyperplane such that $r^{m} \in L_{n-2}{ }^{*}$, and such that $L_{n-2}{ }^{*}$ is perpendicular to $L_{1}{ }^{*}$. The line $L_{1}{ }^{*}$ and the subspace $L_{n-2}{ }^{*}$ determine a hyperplane $L_{n-1}{ }^{*}$. Since $S \cdot L_{n-1}{ }^{*}$ is connected, and since $q$ and $p^{m}$ lie on opposite sides of $L_{n-2}{ }^{*}$ in $L_{n-1}{ }^{*}$, we have $S \cdot L_{n-2}{ }^{*} \neq 0$. Let $s^{m} \in S \cdot L_{n-2}{ }^{*}$. Since $L_{n-2}{ }^{*}$ $C L_{n-1}{ }^{+}$, then $\mathrm{J}^{m} \in L_{n-1}{ }^{+}$. Since by construction $\delta\left(q, y^{m}\right) \rightarrow \infty$ as $m \rightarrow \infty$, $p^{m}$ can be chosen so that $\delta\left(q, p^{m}\right) \rightarrow \infty$ as $m \rightarrow \infty$. Since as $p^{m} \rightarrow \infty$ the line $L_{1}^{*}$ approaches parallelism to $L_{n-1}{ }^{+}, \delta\left(q, r^{m}\right) \rightarrow \infty$ as $p^{m} \rightarrow \infty$. Since $L_{n-2}{ }^{*}$ is perpendicular to $L_{1}{ }^{*}, \delta\left(q, s^{m}\right) \geqq \delta\left(q, r^{m}\right)$. Hence we have $\delta\left(q, s^{m}\right) \rightarrow \infty$ as $m \rightarrow \infty$. Since $s^{m} \in L_{n-2}{ }^{*} C L_{n-1}{ }^{+}$for all $m$, the set $S \cdot L_{n-1}+$ is unbounded. This is a contradiction of hypothesis. Thus $S$ 
is bounded. Since the connectedness of $S$ is well known, Theorem 3 is proved.

2. A characterization of star-like sets. Aumann [1] has characterized compact convex sets by means of properties on linear sections. Also Liberman [4] has made another characterization by placing properties on the set itself and also on its supporting planes. The following theorem, while restricted to two-dimensional sections, yields, as far as it goes, a generalization of Aumann's result, for convexity is replaced by the weaker concept of star-likeness, and boundedness of the set is removed. Note that in Theorem 5 no hypotheses are placed on the set $S$ itself. The following definition is a standard one. Refer to Brunn [2].

Definition. $A$ set $S$ is star-like with respect to a point $a \in S$ if each straight line through a intersects $S$ in a connected set.

In order to characterize star-like sets by linear sections the following definition of simply-connectedness in the plane is especially useful.

Definition. A connected plane set $U$ is simply connected if each component of the complement of $U$ is unbounded.

TheOREM 4 . A closed set $S$ in $R_{n}(n \geqq 3)$ is star-like with respect to a point $a \in S$ if and only if the following conditions hold.

(1) Each two-dimensional linear section of $S$ through the point $a$ is a simply connected, generalized continuum.

(2) For each point $q \in S$, there exists a constant $M>0$, such that each two-dimensional linear section containing $a$ and $q$ contains a continuum joining $a$ and $q$ of diameter less than $M$.

The necessity is immediate. In particular for condition (2) note that $M$ can be any number greater than the distance $\delta(a, q)$.

Sufficiency PRoof. Suppose $S$ is not a star with respect to the point $a$. Then since $S$ is closed, there exist distinct points $b \in S, c \in S$, such that $\delta(a, c)=\delta(a, b)+\delta(b, c)$, and such that the open line segment $L_{1}$ between $b$ and $c$ is not in $S$. Consider any three-dimensional hyperplane $L_{3}$ such that $L_{1} \subset L_{3}$. Choose a coordinate system $(x, y, z)$ in $L_{3}$ so that $L_{1}$ is contained in the $x$-axis. Let $L_{2}{ }^{\theta+} C L_{3}$ be an open half-plane with the $x$-axis as an axis, whose directed normal makes a directed angle $\theta$ with the positive $z$-axis. Also suppose that $0 \leqq \theta \leqq \pi$. Let $L_{2}{ }^{\theta}$ be the plane containing $L_{2}{ }^{\theta+}$, and define ${L_{2}}^{\theta-} \equiv L_{2}{ }^{\theta}-\bar{L}_{2}{ }^{\theta+}$.

Designate the component of the complement of $S_{2}{ }^{\theta} \equiv S \cdot L_{2}{ }^{\theta}$ which contains $L_{1}$ by $C_{2}{ }^{\theta}$. Since $S_{2}{ }^{\theta}$ is a generalized continuum, the boundary of $C_{2}{ }^{\theta}$ is a connected set [6, p. 117]. By a theorem in the plane [5, p. $203 ; 6$, p. 108], the set $C_{2}{ }^{\theta}-L_{1}$ is the sum of two mutually exclusive 
open connected sets $D_{2}{ }^{\theta+}$ and $D_{2}{ }^{\theta-}$, and $L_{1}$ is a subset of the boundary of each of these sets. The set $D_{2}{ }^{\theta+}$ corresponds to $L_{2}{ }^{\theta+}$ in the sense that for any point $r \in L_{1}$, there exists a circle $R_{2} \subset L_{2}{ }^{\theta}$ with center at $r$ such that $D_{2}{ }^{\theta+} \cdot R_{2} \subset L_{2}{ }^{\theta+}$ and $D_{2}{ }^{\theta-} \cdot R_{2} \subset L_{2}{ }^{\theta-}$.

Hypotheses (1) and (2) imply that one and only one of the sets $D_{2}{ }^{\theta+}$ and $D_{2}{ }^{\theta-}$ is unbounded. Furthermore, the bounded set, say $D_{2}{ }^{\theta+}$, is of diameter less than $M$. This is due to the fact that $D_{2}{ }^{\theta+} \subset Q$, where $Q$ is a set enclosed by the closed line segment $(a, c)$ and by the subcontinuum in $S_{2}{ }^{\theta}$ of diameter less than $M$ which joins $a$ and $c$. Clearly $Q$ is of diameter less than $M$, whence $D_{2}{ }^{\theta+}$ is of diameter less than $M$, when it is bounded.

Remark. The set of angles $\{\alpha\}$ for which $D_{2}{ }^{\alpha+}$ is bounded is closed.

To prove this let $L_{2}{ }^{\alpha_{i}} \rightarrow L_{2}{ }^{\alpha}$ as $\alpha_{i} \rightarrow \alpha$, and suppose $D_{2}{ }^{\alpha_{i}+}$ are bounded and that $D_{2}{ }^{\alpha+}$ is unbounded. Choose points $r \in L_{1}$, and $s \in D_{2}{ }^{\alpha+}$ such that the distance $\delta(r, s)>M$. Since $D_{2}{ }^{\alpha+}$ is arcwise connected, let $A \subset D_{2}{ }^{\alpha+}$ be a simple arc joining $r$ and $s$, so that $A \cdot S_{2}{ }^{\alpha}=0$. Rotate $A$ rigidly in $L_{3}$ about $L_{1}$ so that $A^{\alpha_{i}} C L_{2} \alpha_{i}$ is a congruent image of $A$. By virtue of the preceding paragraph, $D_{2}{ }^{\alpha_{i}+}$ are all of diameter less than $M$. Since $A^{\alpha_{i}} \cdot S_{2} \alpha_{i} \neq 0$, since $A^{\alpha_{i}} \cdot S_{2}{ }^{\alpha_{i}}$ are uniformly bounded, and since $S$ is closed, we have $A \cdot S_{2}^{\alpha} \neq 0$. This is a contradiction; hence the remark holds. In exactly the same way, the set of angles $\{\beta\}$ for which $D_{2}{ }^{\beta-}$ is bounded is closed. Since the two closed sets $\{\alpha\}$ and $\{\beta\}$ cover the continuum $0 \leqq \theta \leqq \pi$, they have a value in common. Hence there exists a plane $L_{2}{ }^{\phi}, 0 \leqq \phi \leqq \pi$, such that each $D_{2}{ }^{\phi-}$ and $D_{2}{ }^{\phi+}$ is bounded. But in this case $C_{2}{ }^{\phi}$ would be bounded, and $S_{2}{ }^{\phi}$ would not be simply connected. Hence Theorem 4 is proved.

CoRollary 4.1. Let $S$ be a compact set in $R_{n}$ ( $\left.n \geqq 3\right)$. The set $S$ is a star with respect to a point $a \in S$ if and only if condition (1) in Theorem 4 holds.

Compactness of $S$ and condition (1) imply condition (2). Hence Corollary 4.1 follows from Theorem 4.

THEOREM 5. Let $S$ be any set in $R_{n}(n \geqq 3)$. The set $S$ is a closed convex set if and only if conditions (1) and (2) in Theorem 4 hold for all points $a \in S$.

The necessity is obvious. To prove the sufficiency note that Theorem 1 implies that $S$ is closed. Hence by Theorem $4, S$ is star-like with respect to all points of $S$. Thus by definition $S$ is convex.

3. A theorem in linear spaces. The results of Theorem 3 can be generalized to hold in a normed, linear, metric space $\mathcal{A}$. A hyperplane 
$L$ in $\mathcal{H}$ is defined to be the set $\{x\}$ which satisfies an equation $f(x)=c$, where $f(x)$ is a linear functional, and where $c$ is a real constant. A linear section of $S$ with $L$ is the set $S \cdot L$.

THEOREM 6. Let $S$ be any set in a normed linear metric space $\mathcal{X}$. If each linear section of $S$ is bounded and connected, then $S$ is bounded and connected.

Proof. Consider two independent linear functionals $f_{1}(x)$ and $f_{2}(x)$ defined on $\mathcal{X}$. Let $T$ be a transformation of the type

$T$ :

$$
\xi_{1}=f_{1}(x), \quad \xi_{2}=f_{2}(x) .
$$

This transformation maps $S$ in $\mathcal{X}$ into a set $S_{2}$ in the plane $R_{2}$. Any linear section $S_{2} \cdot L_{1}$ determined by the line $L_{1}, \alpha \xi,+\beta \xi_{2}=\gamma$ corresponds by $T$ to the section $S \cdot L$ where $L$ is defined by $\alpha f_{1}(x)+\beta f_{2}(x)=\gamma$. Since $T$ is linear (additive and continuous), and since by hypothesis $S \cdot L$ is connected and bounded, it follows that the linear section $S_{2} \cdot L_{1}$ is connected and bounded. Hence by Theorem 3 with $n=2$, the set $S_{2}$ is bounded. Thus each functional $f_{1}(x)$ and $f_{2}(x)$ is bounded for all $x$ in $S$. Since $f_{1}(x)$ was an arbitrary linear functional, independent of $f_{2}(x)$, we have shown that all linear functionals defined on $\mathcal{X}$ are bounded on $S$. Hence by a classical theorem of uniform boundedness [3], the set $S$ is bounded. Since the connectedness is well known, Theorem 6 has been established. It should be noted that in light of Theorem 6 the proof for Theorem 3 need only have been given for $n=2$; however, since the proof for $n$ dimensions was not appreciably longer, an elementary proof independent of the abstract boundedness theorem seemed desirable.

4. Concluding remarks. It should be observed that in Theorems 1-3 one cannot delete connectedness entirely, for then the theorems in general are no longer true. Theorem 5 has a preferred form since no hypotheses are placed on $S$ itself. Theorem 4 needs to be formulated so as to hold for $(n-r)$-dimensional sections. This problem is still unsolved. It should be noticed in dealing with non-compact sets that the complement of an unbounded convex set or of an unbounded star need not be connected. Hence conditions on the complement necessary to yield a characterization take on a different form than those given by Aumann [1]. The author wishes to express his gratitude to his colleagues, Professor R. H. Sorgenfrey, Professor W. T. Puckett, and Professor M. Zorn who have made helpful suggestions.

\section{BIBLIOGRAPHY}

1. George Aumann, On a topological characterization of compact convex point sets, 
Ann. of Math. vol. 37 (1936) pp. 443-447.

2. H. Brunn, Über Kerneigebiete, Math. Ann. vol. 73 (1913) pp. 436-440.

3. Nelson Dunford, Uniformity in linear spaces, Trans. Amer. Math. Soc. vol. 44 (1938) pp. 305-354.

4. I. Liberman, On some characteristic properties of convex sets, Rec. Math. (Mat. Sbornik) N.S. vol. 13 (1943) pp. 239-262. See also Mathematical Reviews vol. 6 (1945) p. 184.

5. R. L. Moore, Foundations of point set theory, Amer. Math. Soc. Colloquium Publications, vol. 13, 1932.

6. M. H. A. Newman, Elements of the topology of plane sets of points, Cambridge University Press, 1939.

University of California at los Angeles

\section{THE SPACE $L^{\omega}$ AND CONVEX TOPOLOGICAL RINGS}

\section{RICHARD ARENS}

1. Introduction. The motive for investigating the class $L^{\omega}$ of functions belonging to all $L^{p}$-classes has no measure-theoretic origin: it was our desire to discover whether or not in every convex metric ring $^{1} R$ one could find a system $\{U\}$ of convex neighborhoods of 0 having the property that $f, g \in U$ implies $f g \in U$. We show here that $L^{\omega}$ has no proper convex open set $U$ containing 0 and satisfying the relation $U U \subset U$, thus supplying the desired counter-example.

The significance of neighborhood systems of the type $\{U\}$ described above is made somewhat clearer by a proof that they insure the existence and continuity of entire functions (for example, the exponential function) on the topological ring $R$.

Such neighborhood systems $\{U\}$ are always present in rings of continuous real-valued functions over any space, provided that convergence means uniform convergence on compact sets.

We also consider the relation of $L^{\infty}, L^{\omega}$, and the $L^{p}$-classes, since $L^{\omega}$ does not seem ever to have been discussed as a topological and algebraic entity.

2. Notation and elementary facts. Let us consider measurable functions defined on $[0,1]$. For $p \geqq 1$ we shall consistently employ the usual notation

Received by the editors June 26, 1946.

1 More precisely, metrizable, convex, complete topological linear algebra. For these one requires continuity in both ring operations and scalar multiplication. It will appear that $L^{\omega}$ has these properties. 